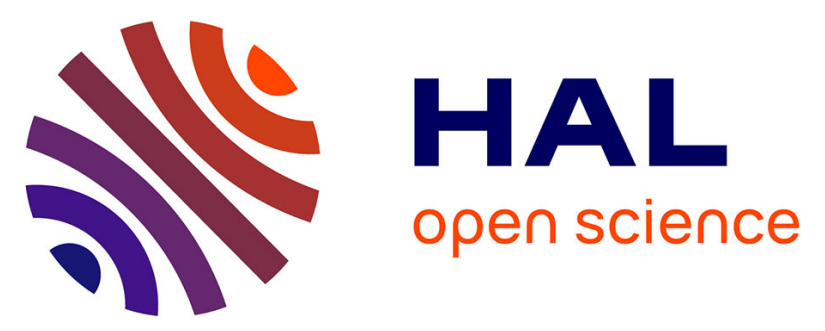

\title{
Effets de l'application de cuivre et de zinc en sol volcanique sur les teneurs du sol et d'un ray-grass anglais en ces deux oligo-éléments
}

Jean Dejou, François-Xavier Montard, Michel Lamand, Jacques Bellanger, Georges Baucher, Olivier Jourdan, Bernard Pons

\section{To cite this version:}

Jean Dejou, François-Xavier Montard, Michel Lamand, Jacques Bellanger, Georges Baucher, et al.. Effets de l'application de cuivre et de zinc en sol volcanique sur les teneurs du sol et d'un ray-grass anglais en ces deux oligo-éléments. Agronomie, 1985, 5 (9), pp.841-850. hal-00884820

\section{HAL Id: hal-00884820 \\ https://hal.science/hal-00884820}

Submitted on 1 Jan 1985

HAL is a multi-disciplinary open access archive for the deposit and dissemination of scientific research documents, whether they are published or not. The documents may come from teaching and research institutions in France or abroad, or from public or private research centers.
L'archive ouverte pluridisciplinaire HAL, est destinée au dépôt et à la diffusion de documents scientifiques de niveau recherche, publiés ou non, émanant des établissements d'enseignement et de recherche français ou étrangers, des laboratoires publics ou privés. 


\title{
Effets de l'application de cuivre et de zinc en sol volcanique sur les teneurs du sol et d'un ray- grass anglais en ces deux oligo-éléments
}

\author{
Jean DEJOU, François-Xavier de MONTARD, Michel LAMAND $\left({ }^{*}\right)$ \& Jacques BELLANGER $\left({ }^{*}\right)$ \\ avec la collaboration technique de Georges BAUCHER, Olivier JOURDAN \& Bernard PONS \\ I.N.R.A., Station d'Agronomie, 12, av. du Brézet, F 63039 Clermont-Ferrand. \\ (*) I.N.R.A., Laboratoire des Maladies nutritionnelles, CRZV de Theix, F 63112 Ceyrat.
}

\begin{abstract}
RÉSUMÉ
Un essai avec apport en couverture, pendant 3 années consécutives, de $\mathrm{Cu}$ et de $\mathrm{Zn}$ sous forme de sulfates, a été installé en sol volcanique de l'Auvergne. Cultivé avec un ray-grass anglais, il a été suivi pendant 8 ans avec plusieurs fertilisations différentes.

Si l'apport des 2 oligo-éléments ne détermine pas d'accroissement de rendement du fourrage comparativement à une fertilisation N P K Mg, il contribue à l'enrichissement du sol, insuffisamment pourvu au départ de l'essai. La qualité des fourrages, carencés à la fois en $\mathrm{Cu}$ et $\mathrm{Zn}$, est améliorée, mais cette amélioration est acquise plus rapidement pour $\mathrm{Zn}$ que pour $\mathrm{Cu}$; par ailleurs, l'apport d'une fumure associée $\mathrm{Cu}+\mathrm{Zn}$ s'avère plus efficace qu'une application séparée des 2 oligo-éléments.
\end{abstract}

Mots clés additionnels : Fourrages, carences en $\mathrm{Cu}$ et $\mathrm{Zn}$, bilans $\mathrm{Cu}$ et $\mathrm{Zn}$.

\begin{abstract}
Effect of surface application of copper and zinc to a volcanic soil on the content of these elements in soil and harvested rye-grass.

An experiment with surface addition, during three consecutive years, of $\mathrm{Cu}$ and $\mathrm{Zn}$ as sulfates, has been carried out on a volcanic soil of the Auvergne. Cultivated with perennial rye-grass, it has been followed over eight years with several different fertilizer regimes.

While the supply of the two oligo-elements did not increase hay yields by comparison with $\mathrm{N} \mathrm{P} \mathrm{K} \mathrm{Mg}$ fertilization, it did contribute to enriching the soil, which was deficient at the beginning of the experiment. The quality of the hay, previously deficient in both $\mathrm{Cu}$ and $\mathrm{Zn}$, was also improved, but this improvement was obtained more rapidly for $\mathrm{Zn}$ than for $\mathrm{Cu}$. However, $\mathrm{Cu}+\mathrm{Zn}$ supplied simultaneously was more effective than separate applications of the two trace-elements.
\end{abstract}

Additional key words : Forage, deficiencies for $\mathrm{Cu}$ and $\mathrm{Zn}, \mathrm{Cu}$ and $\mathrm{Zn}$ balances.

\section{INTRODUCTION}

L'enquête conduite en France sur les oligo-éléments (Bellanger et al., 1973 ; LAMAND \& Perigaud, 1973 ; PERIGAUd \& LAMAND, 1973 ; DE MONTARD \& PERIGAUD, 1975) a montré la grande fréquence des subcarences en cuivre et en zinc dans les fourrages de la plupart de nos régions. L'insuffisance en ces 2 éléments est particulièrement accusée dans les sols volcaniques de l'Auvergne sur les versants les mieux arrosés, souvent très productifs. Il en résulte l'apparition fréquente de graves symptômes cliniques sur les troupeaux bovins (LAMAND, 1970, 1971, 1976).
Les sols développés sur basaltes dans le domaine I.N.R.A. du Roc à Orcival (Puy-de-Dôme) sont typiques à cet égard. Un essai avec application de cuivre et de zinc en couverture y a été installé en 1975 pour étudier la possibilité d'enrichissement à moyen terme des fourrages d'un ray-grass anglais.

Cette expérimentation a déjà fait l'objet d'une première publication (DEJOU et al., 1980). Le but de ce mémoire est de tracer l'évolution des teneurs des 2 oligo-éléments, à la fois dans le sol et dans les fourrages, durant 8 campagnes consécutives (1975-1982) et d'étudier l'impact respectif des paramètres responsables de cette évolution. 


\section{MATÉRIEL ET MÉTHODES}

\section{A. Culture et sol}

Il s'agit d'un ray-grass anglais, variété "Réveille", semé fin avril 1974 dans un andosol développé sur basalte avec ses caractéristiques physico-chimiques classiques en régions tempérées humides :

- texture limoneuse dominante, surtout dans la zone $0-40 \mathrm{~cm}$ du profil,

- richesse en matières organiques depuis la surface $0-10 \mathrm{~cm}$ où la teneur atteint $15 \mathrm{p} .100$ jusqu'en profondeur avec $6,3 \mathrm{p} .100$ dans le niveau $40-60 \mathrm{~cm}$. Il s'agit en l'occurrence d'un isohumisme atténué,

- $\mathrm{pH}$ acide : 5,2 à 5,6 suivant les horizons,

- densité apparente faible : 0,8 en surface ; 0,7 en profondeur,

- faible saturation du complexe argilo-humique : 22 p. 100 dans l'horizon $0-10 \mathrm{~cm}, 8,5$ p. 100 dans la tranche $40-60 \mathrm{~cm}$,

- teneurs en $\mathrm{Cu}$ extrait par EDTA très faible : $0,9 \mathrm{ppm}$ en surface, $0,7 \mathrm{ppm}$ en profondeur, soit des niveaux très bas (OWEN, 1981),

- teneur en $\mathrm{Zn}$ EDTA plus élevée, mais variable avec la profondeur du profil, depuis $12 \mathrm{ppm}$ dans l'horizon $0-10 \mathrm{~cm}$ à $2,7 \mathrm{ppm}$ dans la tranche $40-50$ $\mathrm{cm}$.

\section{B. Protocole de l'essai}

Il comporte 7 traitements différenciés, répétés en 4 blocs sur des parcelles élémentaires de $5 \times 3=15 \mathrm{~m}^{2}$, soit 26 parcelles, l'un d'eux n'ayant que 2 répétitions :

$$
\begin{array}{ll}
\text { traitement } & 1-\mathrm{NP} \\
& 2-\mathrm{NP} \mathrm{Mg} \\
& 3-\mathrm{NP} \mathrm{Mg} \mathrm{K} \\
& 4-\mathrm{NP} \mathrm{Mg} \mathrm{K}+\mathrm{Cu} \\
& 5-\mathrm{NP} \mathrm{MgK}+\mathrm{Zn} \\
& 6-\mathrm{NP} \mathrm{Mg} \mathrm{K}+\mathrm{Cu}+\mathrm{Zn} \\
& 7-\mathrm{NPK} \quad(2 \text { répétitions seulement) }
\end{array}
$$

Les traitements 1, 2 et 7 ont permis l'étude de l'effet de $\mathrm{Mg}$ et de $\mathrm{K}$ sur la production de la matière sèche (DEJOU \& DE MONTARD, 1982).

\section{Doses des éléments fertilisants apportés}

Chaque année, une fumure minérale a été appliquée en couverture comprenant à l'ha :

- 180 à $270 \mathrm{~kg}$ d' $\mathrm{N}$ de l'ammonitrate 33,5 p. 100 en 3 épandages.

$-100 \mathrm{~kg} \mathrm{P}^{2} \mathrm{O}^{5}$ du superphosphate 25 p. 100.

- $240 \mathrm{~kg} \mathrm{~K}{ }^{2} \mathrm{O}$ du chlorure K $60 \mathrm{p} .100$.

- $100 \mathrm{~kg} \mathrm{MgO}$ de l'oxyde de magnésium 100 p. 100

et les 3 premières années de l'essai (1975 à 1977) :

- 7,6 $\mathrm{kg}$ de $\mathrm{Cu}$ du sulfate

- 11,3 $\mathrm{kg}$ de $\mathrm{Zn}$ du sulfate.

\section{Estimation des rendements}

Ils ont été mesurés sur chacune des 3 coupes annuelles du ray-grass à l'aide d'une motofaucheuse de $1,45 \mathrm{~m}$ de large sur une longueur de $5 \mathrm{~m}$, soit une superficie récoltée de $7,25 \mathrm{~m}^{2}$.

\section{E. Techniques analytiques}

$\mathrm{Cu}$ et $\mathrm{Zn}$ ont été dosés à la fois dans le sol et dans la plante.

- Pour le sol, on a retenu un échantillon moyen représentatif des parcelles correspondant aux divers traitements et cela sur 4 niveaux du profil : $0-10$, $10-20,20-40$ et $40-50 \mathrm{~cm}$.

$\mathrm{Cu}$ et $\mathrm{Zn}$ disponibles au sens de OWEN (1981) sont extraits par EDTA 0,04 M et dosés par spectroscopie d'absorption atomique.

- Les fourrages sont minéralisés par attaque nitroperchlorique ; $\mathrm{Cu}$ et $\mathrm{Zn}$ sont ensuite dosés par absorption atomique (BELLANGER, 1971).

\section{RÉSULTATS ET DISCUSSIONS}

\section{A. Rendements moyens du ray-grass anglais}

Les rendements annuels apparaissent sur la figure 1 . En nous limitant aux effets de $\mathrm{Cu}$ et $\mathrm{Zn}$ (traitements 4, 5 et 6), on constate que, quelle que soit l'année, y compris 1975, 1976 et 1977 où les applications d'oligoéléments ont eu lieu, leur apport ne s'est pas soldé par un accroissement significatif du rendement de la graminée (p.p.d.s. compris entre 0,9 et $1,1 \mathrm{t} / \mathrm{ha} / \mathrm{MS}$ pour $P=0,05)$. Il n'y a donc pas eu d'effet de cette fertilisation pas plus que d'arrière-effet.

Ces données corroborent celles citées par PERIGAUD (1970) concluant à un «effet parfois limité " des engrais cupriques sur la production des fourrages alors que des résultats très positifs sont acquis sur céréales avec des suppléments de récolte pouvant atteindre presque 100 p. 100 (BALLAND, 1983).

\section{B. Evolution du cuivre dans le sol et la plante}

\section{Dans le sol}

Depuis le début de l'essai en 1975 jusqu'à son terme, l'évolution du cuivre (EDTA) dans le sol est très différente suivant le traitement appliqué (fig. 2).

$\mathrm{Au}$ départ, la teneur en $\mathrm{Cu}$ est assez homogène le long du profil et très réduite.

En fin d'essai, on constate que les parcelles non fertilisées en $\mathrm{Cu}$ ont subi une légère diminution de leurs réserves en $\mathrm{Cu}$, par suite de l'absorption de cet élément par le ray-grass sur l'ensemble du profil et de l'exportation des récoltes. La zone $10-20 \mathrm{~cm}$ paraît un peu plus appauvrie que la surface $0-10 \mathrm{~cm}$ où se concentre une grande partie du système racinaire : les racines ont précisément la propriété d'accumuler cet élément (cf. tabl. 3) dont la migration vers l'appareil aérien serait conditionnée par le flux d'azote (JARvis, 1981).

Quand on apporte du $\mathrm{Cu}$ au sol (traitement 4), l'enrichissement est très sensible dans les 10 premiers $\mathrm{cm}$, ce qui confirme d'autres observations effectuées sur fétuque et rapportées par BALLAND (1983). La migration de l'oligo-élément se poursuit encore en profondeur, mais de manière beaucoup plus réduite en diminuant de façon progressive : au-delà de $40 \mathrm{~cm}$, elle est très limitée. 


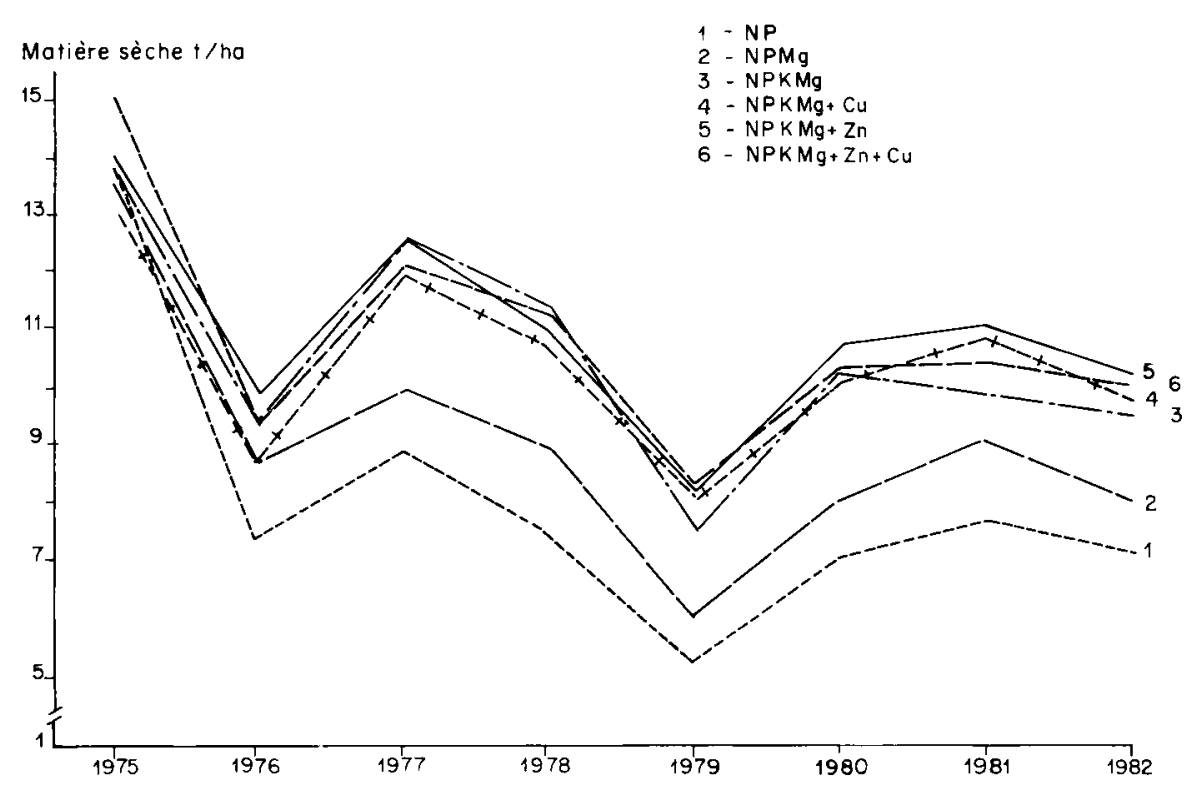

Figure 1

Rendement annuel du ray-anglais suivant les divers traitements.
Total annual yield of perennial rye-grass according to different treatments (dry matter as t.ha $\left.{ }^{1}\right)$.

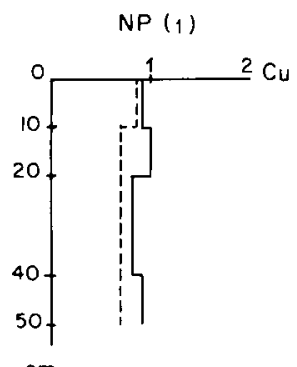

$N P K M g+Z n(5)$

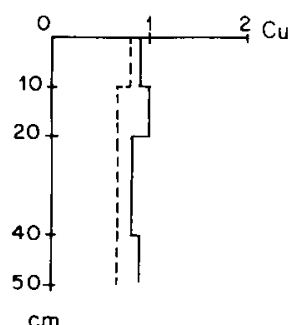

$\mathrm{cm}$

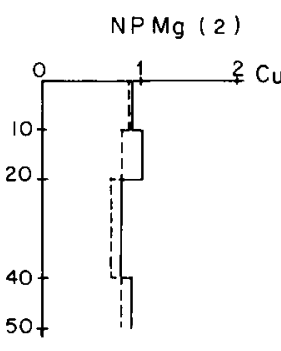

$\mathrm{cm}$

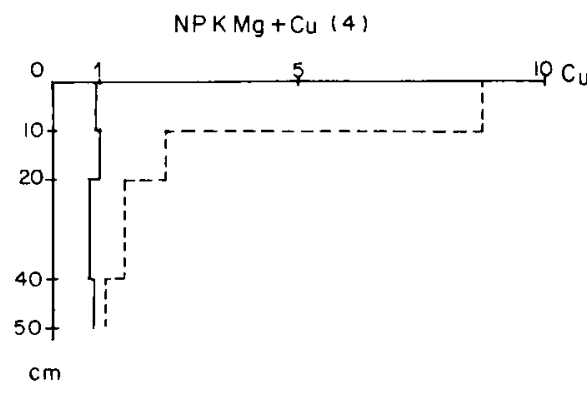

$\mathrm{NPKMg}+\mathrm{Cu}+\mathrm{Zn}(6)$

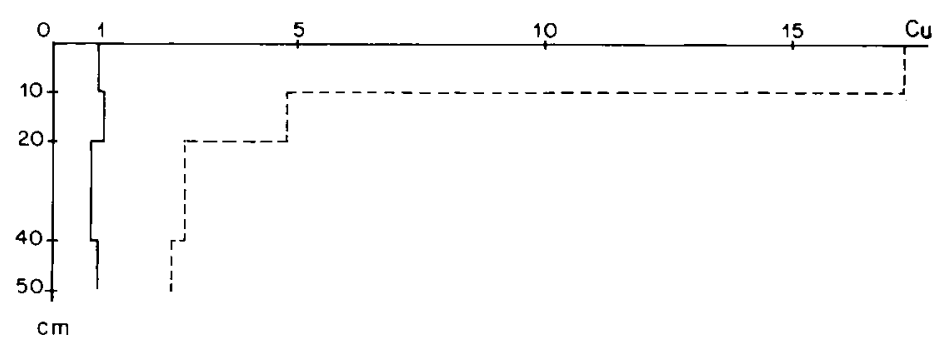

Figure 2

Evolution du CU EDTA en sol volcanique au cours de l'essai fen ppm).

Valeurs initiales (1975)

Valeurs finales (1982).

Mais quand on ajoute à la fois $\mathrm{Cu}$ et $\mathrm{Zn}$ (traitement 6), on constate dans la couche $0-10 \mathrm{~cm}$ du sol un enrichissement en $\mathrm{Cu} 2$ fois supérieur à celui du traitement 4 où $\mathrm{Cu}$ est appliqué seul. Comme précédemment, il diminue en profondeur, tout en restant toujours à un niveau très supérieur. Il y a donc un effet de synergie $\mathrm{Cu}-\mathrm{Zn}$ qu'il est, à vrai dire, difficile d'expliquer. L'hypothèse d'un lessivage plus fort dans le traitement seul semble peu probable dans les sols volcaniques où $\mathrm{Cu}$ est puissamment retenu par les argiles et davantage, semble-t-il dans le cas présent, que par la matière organique pourtant abondante et les allophanes (TRICHET, 1983). Peut-être faut-il invo-
Change in EDTA Cu content in the volcanic soil during the experiment (ppm).

$$
\text { Initial contents (1975). }
$$

Final contents (1982).

quer à la suite de LONERAGAN et al. (1981) la genèse dans le sol de précipités ou de complexes cuivriques peu solubles à partir du sulfate introduit et aussi des ions $\mathrm{PO}^{4} \mathrm{du}$ superphosphate? Leur formation serait moins aisée en présence de $\mathrm{Zn}$.

Finalement, le cuivre EDTA est présent en bien plus grande quantité après une fertilisation au sulfate de cuivre associée à une fertilisation au sulfate de zinc.

\section{Dans la plante}

Les teneurs en $\mathrm{Cu}$ des fourrages subissent des variations très importantes au cours des 8 campagnes de 


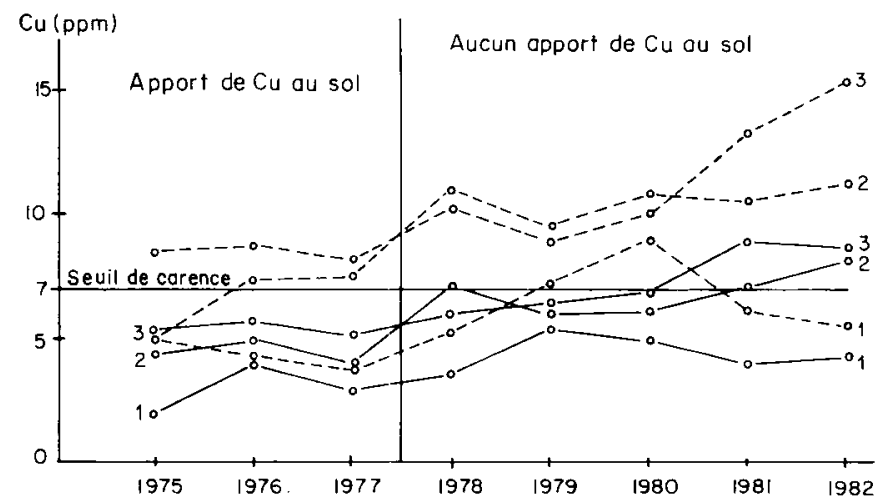

Figure 3

Variation des teneurs en $\mathrm{Cu}$ (ppm/MS) du ray-grass anglais au cours de l'essai (1975-1982).

Variation in $\mathrm{Cu}$ content (ppm of dry matter) in the perennial ryegrass during the experiment (1975-1982).

Moyenne des teneurs des fourrages dans les 5 traitements sans apport de $\mathrm{Cu}\left(n^{\circ} \mathrm{1}, 2,3,5,7\right)$.

Average $\mathrm{Cu}$ contents in hays from the five treatments without $\mathrm{Cu}$ addition $(1,2,3,5$ and 7$)$.

-.----- Moyenne des teneurs des fourrages dans les 2 traitements avec apport de $\mathrm{Cu}\left(n^{o s} 4\right.$ et 6$)$.

Average $\mathrm{Cu}$ contents in hays from the two treatments with $\mathrm{Cu}$ addition (4 and 6).

\section{Remarques}

a) 1,2 et 3 désignent l'ordre des coupes

b) les apports du $\mathrm{Cu}$ au sol ont cessé au printemps 1977

c) la moyenne générale des teneurs en $\mathrm{Cu}$ pour les 8 campagnes de l'essai dans les parcelles sans apport de Cu s'établit respectivement pour les 3 coupes à $4,1-6,2$ et $6,7 \mathrm{ppm}$.

Dans les parcelles avec apport de $\mathrm{Cu}$, elle est de 6,3-9,4 et 10,2 ppm. Les différences des moyennes sont très significatives. Pour $P=0,001$ ( ppds $=1,31-2,27$ et $2,79 \mathrm{ppm} / \mathrm{cm}$ ).

\section{Remarks}

a) $1,2,3=$ successive cuts

b) supplies of $\mathrm{Cu}$ to the soil stopped in spring 1977

c) The overall average contents for the eight years of the experiment in the plots without $\mathrm{Cu}$ addition was respectively in three cuts : 4.1, 6.2 and $6.7 \mathrm{ppm}$, and in the plots with $\mathrm{Cu}$ addition : 6.3 9.4 and $10.2 \mathrm{ppm}$.

The differences between the averages were highly significant at $P=0.001$ (ppds $=1.31,2.27$ and $2.79 \mathrm{ppm} / \mathrm{Cu}$ ).

l'essai selon que cet oligo-élément est apporté ou non dans le sol (fig. 3).

La composition des fourrages sur les parcelles n'ayant pas reçu de $\mathrm{Cu}$ est nettement inférieure au seuil de carence retenu pour les bovins, soit $7 \mathrm{ppm} / \mathrm{MS}$ (COPPENET, $1970 a$ et $b$; LAMAND \& GUEGUEN, 1978), surtout pour la $1^{\text {re }}$ coupe, la plus importante puisqu'elle représente environ 50 p. 100 de la production annuelle globale. Les teneurs des $2^{\mathrm{e}}$ et $3^{\mathrm{e}}$ coupes sont toujours supérieures à celles de la $1^{\text {re }}$ coupe, par suite d'un moindre effet de dilution; à la fin de l'essai, les fourrages sont même correctement pourvus en $\mathrm{Cu}$.

Il est remarquable par ailleurs de constater, du moins de 1975 à 1980, une similitude de l'évolution des teneurs en $\mathrm{Cu}$ dans les différentes coupes, traduite par un certain parallélisme des courbes. Par contre, celui-ci s'estompe à partir de 1980 pour les dernières campagnes avec une évolution divergente selon la saison : $1^{\text {re }}$ coupe de plus en plus pauvre, en corollaire semble-t-il, d'un accroissement en 1980, 1981 et 1982 de la dose d'azote apportée en fin d'hiver ; par contre, augmentation en $2^{\mathrm{e}}$ et $3^{\mathrm{e}}$ coupes.
L'apport de $\mathrm{Cu}$ au sol détermine une augmentation des teneurs de cet élément dans les fourrages, mais de façon très différente suivant les coupes. Les teneurs de la $1^{\text {re }}$ coupe sont toujours supérieures et d'une manière hautement significative $(P=0,001)$ à celles des parcelles n'ayant pas reçu d'apport, quelle que soit l'année de récolte. Mais, jusqu'en 1977, elles sont nettement inférieures au seuil de carence. Ce n'est qu'à partir de cette date, où pourtant les applications au sol ont cessé, que l'on assiste à une croissance continue de la teneur en $\mathrm{Cu}$ des fourrages qui devient maximale en 1980. Ensuite, celle-ci baisse à nouveau et on revient au-dessous de $7 \mathrm{ppm}$ corrélativement avec la variation de la teneur en $\mathrm{N}$ de la plante (fig. 4).

Par conséquent, il y a au départ un transfert très lent de l'oligo-élément depuis le sol, où il est énergiquement retenu, vers la plante, de sorte que la composition des fourrages est d'abord peu affectée par l'apport cuivrique. Par la suite, un arrière-effet très positif s'amorce à partir de 1978, atteint le maximum d'amplitude en 1980 pour décroître ensuite.

Pour les $2^{\mathrm{e}}$ et $3^{\mathrm{e}}$ coupes, la cession du $\mathrm{Cu}$ à la plante est beaucoup plus rapide que pour la $1^{\text {re }}$ coupe. Tous les fourrages, à l'exception de la $2^{\mathrm{e}}$ coupe de 1975 , ont dépassé le seuil critique de carence et sont donc correctement pourvus. Là aussi, l'arrière-effet de l'apport de $\mathrm{Cu}$ détermine une amélioration très hautement significative de la qualité des fourrages $(P=0,001)$, mais qui, à l'inverse de la $1^{\text {re }}$ coupe, se poursuit et s'accentue même à la fin de l'essai. Les résultats obtenus pour les traitements avec ou sans $\mathrm{Cu}$ sont dans l'ensemble homogènes avec des coefficients de variation pour les diverses teneurs oscillant entre 5,6 et 13 p. 100 suivant les années.

La synergie $\mathrm{Cu}-\mathrm{Zn}$ observée au niveau du sol ne se manifeste pas sur les fourrages avant 1978. Elle apparaît d'une manière significative pour $P=0,05$ sur la $2^{\mathrm{e}}$ coupe de 1979 et sur toutes les coupes en 1980 . Le phénomène est d'ailleurs plus accusé sur les $2^{\mathrm{e}}$ et $3^{\text {e }}$ coupes. Par contre, elle n'existe plus en 1981 et 1982 ; cette synergie est donc fugace.

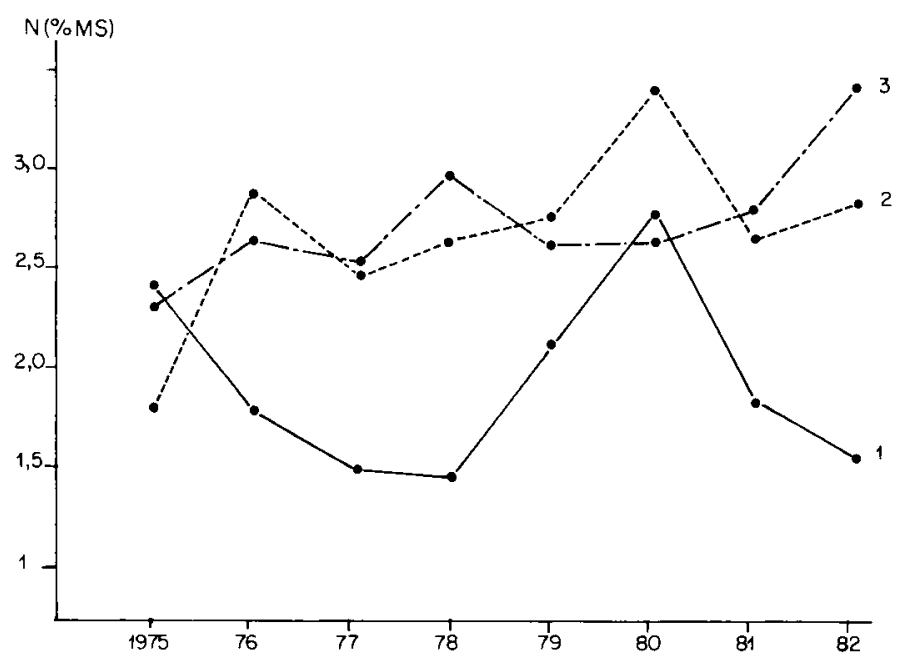

Figure 4

Variation des teneurs en azote des récoltes (movenne par coupe en $\%$ MS de 1975 à 1982). 1, 2 et 3 désignent l'ordre des coupes.

Variation of $N$ content in hay (average for cuts in \% dry matter from 1975 to 1982). 1, 2, 3 : successive cuts. 
Facteurs de variation du cuivre dans les fourrages

Ces divergences entre les teneurs en cuivre des différentes coupes peuvent être expliquées par l'intervention d'un nombre limité de paramètres.

On peut écrire l'équation :

$\mathrm{Cu}(\mathrm{ppm})=2,56+0,098 \mathrm{~F} . \mathrm{Cu}+2,20 \mathrm{~N}$ (plante) $-0,028$ fertilisation $\mathrm{N}$ appliquée $+0,218 \mathrm{Zn}$ appliqué au sol $\pm 2,38$ avec les caractéristiques données dans le tableau 1 pour 95 échantillons.

Le $1^{\text {er }}$ facteur $\mathrm{F}$. $\mathrm{Cu}$ ou « facteur $\mathrm{Cu}$ » est le produit: (nombre d'applications $\mathrm{Cu} \times$ nombre de coupes depuis le dernier apport de $\mathrm{Cu}$ ). On tient compte ainsi des paramètres de la fertilisation cuivrique appliquée dans cet essai. On est frappé de constater que le cuivre devient de plus en plus disponible au cours du temps.

Le $2^{\mathrm{e}}$ facteur est la teneur en $\mathrm{N}$ de la plante dont les variations sont explicitées dans la figure 4 . Il explique 25 p. 100 de la variance totale. Le flux d'azote commande le transfert du cuivre (JARVIS, 1981 ; COPPENET \& SIMON, 1984) et le processus expliquerait le parallélisme observé entre les courbes des figures 3 et 4 , les témoins compris. Par contre, l'acidification du sol constatée ailleurs à la suite de fortes applications d'ammonitrate (SIMON et al., 1983) n'a pu être mise en évidence dans le sol d'Orcival.

Le $3^{\text {e }}$ facteur est la dose d'N incorporée au sol. Il traduit un effet de dilution lié à une plus forte production de matière sèche. La croissance entraîne une diminution conjointe de l'azote et du cuivre.

Le $4^{\mathrm{e}}$ facteur, qui est le nombre d'apports de $\mathrm{Zn}$ au sol, n'a que peu d'incidence sur la teneur en $\mathrm{Cu}$ des fourrages. Avec la fertilisation zincique, le Cu EDTA est plus abondant et il est transféré dans les feuilles parallèlement à la migration de l'azote.
TABLEAU 1

Facteurs de variation des teneurs en $\mathrm{Cu}$ des fourrages.

Factors of variation of $\mathrm{Cu}$ content in hay.

\begin{tabular}{|c|c|c|c|c|}
\hline $\begin{array}{c}\text { Variables } \\
\text { indépendantes }\end{array}$ & $\begin{array}{l}\text { Coefficient } \\
\text { de régression }\end{array}$ & $\begin{array}{c}\text { Ecart-type } \\
\text { du coefficient }\end{array}$ & Test F & $\begin{array}{c}\text { Variance } \\
\text { expliquée } \%\end{array}$ \\
\hline 1) $\mathrm{F} \cdot \mathrm{Cu}$ & $+0,098$ & $\pm 0,008$ & 12,7 & 49,4 \\
\hline $\begin{array}{l}\text { 2) } \mathrm{N} \% \text { de la } \\
\text { plante }\end{array}$ & $+2,20$ & $\pm 0,23$ & 9,6 & 73,9 \\
\hline $\begin{array}{l}\text { 3) Fertilisation } \\
\mathrm{N} \mathrm{du} \mathrm{sol}\end{array}$ & $-0,028$ & $\pm 0,005$ & $-5,3$ & 79,4 \\
\hline $\begin{array}{l}\text { 4) Fumure } \mathrm{Zn} \\
\text { du sol }\end{array}$ & $+0,218$ & $\pm 0,09$ & 2,5 & 80,8 \\
\hline
\end{tabular}

\section{Evolution du zinc dans le sol et la plante}

\section{Dans le sol}

L'évolution du Zn dans le sol présente de notables différences avec celle du $\mathrm{Cu}$ (fig. 5).

Au départ de l'essai, la répartition du $\mathrm{Zn}$ dans le sol, contrairement à celle du $\mathrm{Cu}$, n'est pas homogène, sa concentration la plus importante se situant en surface dans la zone $0-10 \mathrm{~cm}$. La diminution est sensible de 10 à $40 \mathrm{~cm}$ et très accusée au-delà de cette limite pour atteindre $2,7 \mathrm{ppm}$.

En fin d'essai, dans les parcelles non fertilisées (1), (2) et (4), le ray-grass a puisé le $\mathrm{Zn}$ dans l'ensemble du niveau $0-40 \mathrm{~cm}$.

Quand on apporte du Zn au sol (traitement 5), l'enrichissement est très sensible, mais seulement dans les 10 premiers $\mathrm{cm}$, contrairement au $\mathrm{Cu}$, et l'on constate même une diminution du taux initial de 10 à $40 \mathrm{~cm}$ par suite de l'activité racinaire.
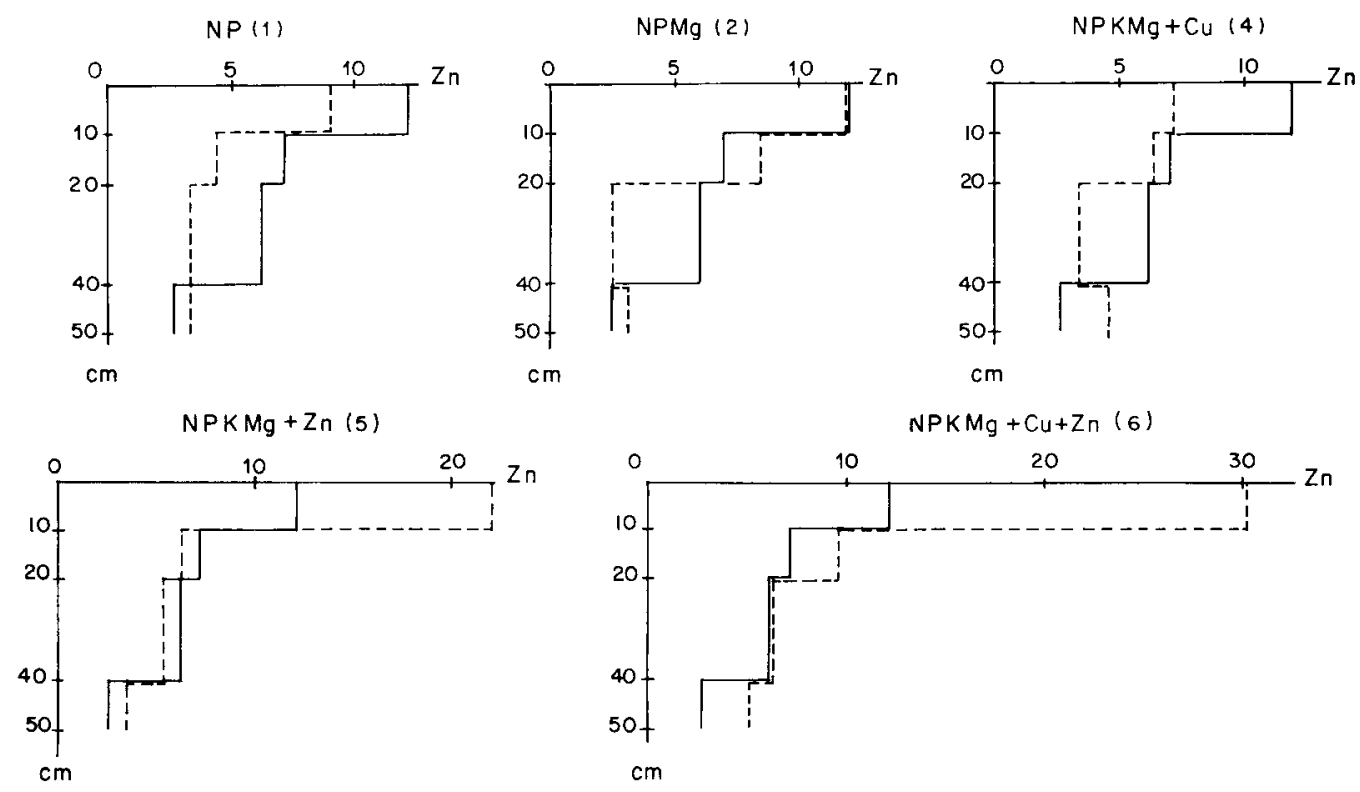

Figure 5

Evolution du Zn EDTA en sol volcanique au cours de l'essai (en ppm). 
Quand les oligo-éléments $\mathrm{Cu}$ et $\mathrm{Zn}$ sont appliqués ensemble (traitement 6), un effet de synergie $\mathrm{Zn}-\mathrm{Cu}$ est enregistré : le taux de $\mathrm{Zn}$ dans l'horizon $0-10 \mathrm{~cm}$ atteint $31 \mathrm{ppm}$ contre $22 \mathrm{ppm}$ avec le $\mathrm{Zn}$ incorporé seul. L'enrichissement se poursuit faiblement dans le niveau $10-20 \mathrm{~cm}$, très peu dans $20-40 \mathrm{~cm}$ et est très sensible encore days $40-50 \mathrm{~cm}$. La migration de $\mathrm{Zn}$ dans le sol est dónc différente de celle du cuivre.

\section{Dans la plante}

On note certaines analogies avec le comportement du cuivre, mais surtout des différences très nettes (fig. 6).

Tous les fourrages récoltés sur les parcelles n'ayant pas reçu d'apport de Zn à l'exception des 3 coupes de 1981 et de 1982, sont insuffisamment pourvus en cet élément ; les teneurs se situent au-dessous du seuil de carence de $40 \mathrm{ppm} / \mathrm{MS}$ pour les bovins (LAMAND, 1971).

L'apport du Zn au sol augmente la qualité du fourrage dès la $1^{\text {re }}$ année, mais les teneurs des 3 coupes restent proches du seuil de carence. Ensuite, l'amélioration est très importante jusqu'en 1980, surtout pour les $2^{\mathrm{e}}$ et $3^{\mathrm{e}}$ coupes, très largement pourvues en cet élément, malgré des fluctuations très accusées d'une année à l'autre. C'est en 1980 que les teneurs en $\mathrm{Zn}$ sont les plus fortes, quelle que soit la coupe. Ensuite, elles diminuent progressivement. Mais dans tous les cas, les teneurs des parcelles fertilisées sont très significativement différentes $(\mathrm{P}=0,001)$ de celles qui n'ont pas reçu d'apport. Bien que le zinc migre peu, il reste disponible pour l'absorption, et surtout son transfert vers les parties aériennes est plus aisé que celui du cuivre.

Les arrières-effets des fertilisations zinciques appliquées de 1975 à 1977 sont massifs et prolongés jusqu'en 1982 comme en témoignent les résultats indiqués dans le tableau 2.

Bien que la partie supérieure du sol $(0-10 \mathrm{~cm})$ soit encore nettement enrichie en $\mathrm{Zn}$ extractible par EDTA, l'effet de la fertilisation zincique sur les teneurs des fourrages diminue fortement à la fin de l'essai.

\section{Facteurs de variation $d u \mathrm{Zn}$ dans les fourrages}

Tout comme pour $\mathrm{Cu}$, la teneur en $\mathrm{Zn}$ (en ppm) des fourrages est fonction d'un certain nombre de paramètres, et on peut écrire l'équation suivante dont les caractéristiques sont précisées dans le tableau 3 :

$\mathrm{Zn}(\mathrm{ppm})=0,106+8,01$ nombre d'apports de $\mathrm{Zn}$

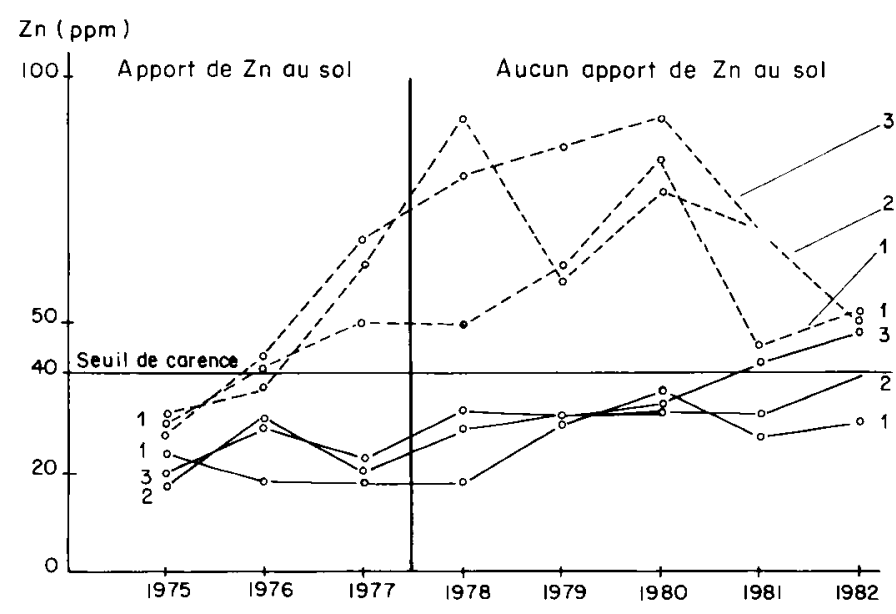

Figure 6

Variations des teneurs en $Z n$ (en ppm) du ray-grass anglais au cours de l'essai (1975-1982).

Variation in $Z n$ content (ppm of dry matter) in the perennial ryegrass during the experiment (1975-1982).

Moyenne des teneurs des fourrages dans les 5 traitements sans apport de $\mathrm{Zn}\left(n^{o s} 1,2,3,4\right.$ et 7$)$.

Average $\mathrm{Zn}$ contents in hay from the five treatments without $\mathrm{Zn}$ addition $(1,2,3,4,7)$.

------ Moyenne des teneurs des fourrages dans les 2 traitements avec apport de $\mathrm{Zn}\left(n^{\text {os }} 5\right.$ et 6$)$.

Average $\mathrm{Zn}$ contents in hay from the two treatments with $\mathrm{Zn}$ addition.

\section{Remarques}

a) 1,2 et 3 désignent l'ordre des coupes

b) les apports de Zn au sol ont cessé au printemps 1977

c) la moyenne générale des teneurs en $Z n$ pour les 8 campagnes de l'essai dans les parcelles sans apport de $\mathrm{Zn}$ s'établit respectivement pour les 3 coupes à 26,4-30,5 et 31,8 ppm.

Dans les parcelles avec apport de Zn, elle est de 52,5-64,5 et $59,2 \mathrm{ppm}$. Les différences des moyennes sont très significatives pour $P=0,001$ (ppds $=22,4-20,7$ et 17,2 ppm).

Remarks

a) $1,2,3:$ successive cuts

b) Supplies of $\mathrm{Zn}$ to the soil stopped in spring 1977

c) The overall average content $\mathrm{Zn}$ for the eight years of the experiment in the plots without $\mathrm{Zn}$ addition was respectively in three cuts : 26.4, 30.5 and $31.8 \mathrm{ppm}$, and in the plots with $\mathrm{Zn}$ addition : $52.5,64.5$ and $59.2 \mathrm{ppm}$. The differences between the averages were highly significant at $P=0.001$ (ppds $=22.4,20.7$ and $17.2 \mathrm{ppm}$ ).

au sol $+10,6 \mathrm{~N}$ p. 100 des fourrages $+1,69$ (nombre d'apports de $\mathrm{Zn} \times$ nombre de coupes) $-0,10$ nombre d'apports de $\mathrm{Zn} \times$ (nombre de coupes) $^{2}+0,18$ (nombre d'apports de $\mathrm{Cu} \times$ nombre de coupes $)+16,4$.

Le $1^{\text {er }}$ facteur rend compte des effets cumulatifs des 3 apports de $\mathrm{Zn}$ successifs (1975, 76 et 77 ).

TABLEAU 2

Teneurs en $\mathrm{Zn}$ (ppm) des fourrages dans les parcelles fertilisées. Accroissement relatif de ces teneurs en comparaison avec celles des parcelles n'ayant pas reçu d'apport (moyenne des 3 coupes annuelles). $\mathrm{Zn}$ content (ppm) of hay in the fertilized plots and increase by comparison with control (average of three annual cuts).

\begin{tabular}{lrrrrrrrr}
\hline \hline Année & 1975 & 1976 & 1977 & 1978 & 1979 & 1980 & 1981 & 1982 \\
\hline $\begin{array}{l}\text { Teneurs en Zn (ppm) des parcelles ferti- } \\
\text { lisées }\end{array}$ & 28,0 & 40,0 & 60,7 & 74,5 & 69,3 & 84,0 & 61,3 & 52,7 \\
\hline \begin{tabular}{l} 
Accroissement (en \%) relatif \\
\hline
\end{tabular} & +30 & $+48,5$ & +173 & +162 & +122 & +131 & +74 & +33 \\
\hline
\end{tabular}


TABLEAU 3

Facteurs de variation des teneurs en $\mathrm{Zn}$ des fourrages. Factors of variation of $\mathrm{Zn}$ content in hay.

\begin{tabular}{|c|c|c|c|c|c|}
\hline & $\begin{array}{c}\text { Variables } \\
\text { indépendantes }\end{array}$ & $\begin{array}{l}\text { Coefficient } \\
\text { de } \\
\text { régression }\end{array}$ & $\begin{array}{c}\text { Ecart-type } \\
\text { du } \\
\text { coefficient }\end{array}$ & Test $\mathrm{r}$ & $\begin{array}{c}\text { Variance } \\
\text { expliquée } \\
(\%)\end{array}$ \\
\hline 1) & $\begin{array}{l}\text { Nombre d'apports de } \\
\text { Zn au sol }\end{array}$ & 8,01 & 1,45 & 5,54 & 37,0 \\
\hline 2) & $\begin{array}{l}\text { Teneur } \mathrm{N} \% \text { des } \\
\text { fourrages }\end{array}$ & 10,6 & 1,61 & 6,60 & 79,5 \\
\hline 3) & $\begin{array}{l}\text { Nombre d'apports } \mathrm{Zn} \\
\times \text { nombre de coupes }\end{array}$ & 1,69 & 0,35 & 4,87 & \\
\hline 4) & $\begin{array}{l}\text { Nombre d'apports } \mathrm{Zn} \\
\times \text { (nombre de cou- } \\
\text { pes })^{2}\end{array}$ & $-\quad 0,10$ & 0,018 & $-5,56$ & 84,5 \\
\hline 5) & $\begin{array}{l}\text { Nombre d'apports } \mathrm{Cu} \\
\times \text { nombre de coupes }\end{array}$ & $+0,18$ & 0,055 & 3,32 & 86,2 \\
\hline
\end{tabular}

Le $2^{\mathrm{e}}$ facteur indique que le transfert $\mathrm{du} \mathrm{Zn}$ vers l'appareil aérien est, comme celui du $\mathrm{Cu}$, parallèle à celui de l'azote ; comme pour ce dernier élément, la teneur en $\mathrm{Zn}$ diminue avec l'âge de la plante.

Les $3^{\mathrm{e}}$ et $4^{\mathrm{e}}$ facteurs font apparaître l'effet du nombre de coupes depuis le dernier apport sous forme quadratique en interaction avec le nombre d'apports de Zn. Mais cette interaction reste modeste et son influence diminue un an après le dernier épandage.

Le dernier facteur indique un effet significatif de l'apport conjoint de $\mathrm{Zn}$ et de $\mathrm{Cu}$ sur la teneur en $\mathrm{Zn}$ des fourrages.

\section{Essai de bilan global des oligo-éléments $\mathrm{Cu}$ et $\mathrm{Zn}$ en sol volcanique}

\section{Bilans culturaux}

Les bilans culturaux en $\mathrm{Cu}$ et $\mathrm{Zn}$ sont obtenus par comparaison entre les apports et les sorties des 2 oligoéléments.

Les sorties comprennent :

\section{a) Les exportations}

Elles sont calculées pour les 3 coupes annuelles après dosage du $\mathrm{Cu}$ et du $\mathrm{Zn}$ sur un échantillon représentatif, correspondant à chacun des 7 traitements.

\section{b) Les quantités stockées dans les racines}

Elles ont fait l'objet d'une estimation en août 1981 (tabl. 4) à partir d'un échantillonnage effectué dans l'horizon $0-5 \mathrm{~cm}$ du sol où leur densité est la plus forte. Cette évaluation, particulièrement délicate, est sûrement sous-estimée, puisqu'elle est réalisée à une période donnée, alors que le système racinaire est en renouvellement constant.

A la suite de COIC et al. (1974), on constate que les racines stockent chez le ray-grass anglais une quantité considérablement plus forte de $\mathrm{Cu}$ et de $\mathrm{Zn}$ que les parties aériennes, surtout dans les parcelles enrichies où la teneur en $\mathrm{Cu}$ des racines est au moins 30 fois plus élevée que dans les foins de $1^{\text {re }}$ coupe et près de 20 fois plus que dans ceux de $2^{\mathrm{e}}$ et $3^{\mathrm{e}}$ coupes, tandis que celle en $\mathrm{Zn}$ est de 4 à 5 fois supérieure. Cette accumulation n'est absolument pas générale dans le règne végétal (DARTIGUES, 1964). Mais ici, elle est tellement marquée pour $\mathrm{Cu}$ dans les racines des parcelles fertilisées qu'elle dépasse les exportations totales en cet élément pendant toute la durée de l'essai ; par contre, le stock $\mathrm{Zn}$ des racines ne représente que le tiers environ de ces exportations.

Les bilans (tabl. 5) sont largement positifs dans le cas des parcelles fertilisées en $\mathrm{Cu}$ et $\mathrm{Zn}$ et d'autant plus déficitaires dans les autres que le rendement en matière sèche est lui-même plus élevé.

\section{Bilans d'après les analyses de sols}

Les bilans (tabl. 5) ont été établis en analysant les éléments $\mathrm{Cu}$ et $\mathrm{Zn}$ échangeables par la technique EDTA et en calculant les quantités disponibles au début et à la fin de l'essai sur les 50 premiers $\mathrm{cm}$ du profil.

Cette estimation est encore plus délicate que celle des bilans culturaux en raison des hétérogénéités locales de la composition des sols, des points de prélèvements qui ne sont pas rigoureusement les mêmes au départ et à la fin de l'essai, éventuellement de la limitation des observations au niveau $50 \mathrm{~cm}$, enfin de la

TABLEAU 4

Teneurs moyennes des racines et des parties aériennes (tiges + feuilles) du ray-grass anglais en oligo-éléments (en ppm MS). Average micronutrient content of roots and aerial parts (stems and leaves) of perennial rye-grass (ppm DM).

\begin{tabular}{|c|c|c|c|c|c|c|c|}
\hline \multirow[t]{2}{*}{ Traitement } & \multirow{2}{*}{$\begin{array}{c}\text { Poids des racines } \\
\text { en } \mathrm{t} / \mathrm{ha} / \mathrm{MS}\end{array}$} & \multirow{2}{*}{\multicolumn{2}{|c|}{ Racines }} & \multicolumn{4}{|c|}{ Parties aériennes } \\
\hline & & & & & & $2^{\mathrm{e}} \mathrm{e}$ & pes \\
\hline $\begin{array}{c}\text { Nombre d'échantillons } \\
\text { examinés }\end{array}$ & 8 & \multicolumn{2}{|c|}{4} & \multicolumn{2}{|c|}{32} & \multicolumn{2}{|c|}{64} \\
\hline Oligo-éléments & & $\mathrm{Cu}$ & $\mathrm{Zn}$ & $\mathrm{Cu}$ & $\mathrm{Zn}$ & $\mathrm{Cu}$ & $\mathrm{Zn}$ \\
\hline 3) N P K Mg & 6,78 & 13,7 & 92,2 & 3,8 & 26,0 & 6,2 & 29,4 \\
\hline 4) $\mathrm{NPKMg}+\mathrm{Cu}$ & 6,05 & 188,0 & 90,0 & 5,9 & 27,4 & 9,8 & 33,6 \\
\hline 5) $\mathrm{NPKMg}+\mathrm{Zn}$ & 5,35 & 20,7 & 257,0 & 4,4 & 49,1 & 6,5 & 60,1 \\
\hline 6) $\mathrm{NPKMg}+\mathrm{Cu}+\mathrm{Zn}$ & 6,85 & 222,5 & 296,0 & 6,6 & 55,7 & 10,0 & 64,5 \\
\hline
\end{tabular}

Remarques :

1) Les racines ont été prélevées dans la zone $0-5 \mathrm{~cm}$ du sol le 12.8.1981.

2) Pour le dosage des parties aériennes de la plante, les moyennes du tableau sont calculées sur 8 coupes $\times 4$ répétitions $=32$ échantillons en

$1^{\text {re }}$ coupe et 16 coupes $\times 4$ répétitions $=64$ échantillons en $2^{\mathrm{e}}$ et $3^{\mathrm{e}}$ coupes. 
TABLEAU 5

Essais de bilans des oligo-éléments $\mathrm{Cu}$ et $\mathrm{Zn}$ à Orcival (1975 à 1982).

Lignes 1 à 4 : bilans culturaux; lignes 5 à 7 : bilans d'après les analyses du sol de 0 à $50 \mathrm{~cm}$ (technique EDTA). Trials on $\mathrm{Cu}$ and $\mathrm{Zn}$ balances at Orcival.

Lines 1 to 4 : crop balances; lines 5 to $7:$ soil balances, $0-50 \mathrm{~cm}$.

\begin{tabular}{|c|c|c|c|c|c|c|}
\hline Traitements & $\begin{array}{l}\text { NP } \\
\text { (1) }\end{array}$ & $\begin{array}{c}\mathrm{NP} M g \\
\text { (2) }\end{array}$ & $\begin{array}{c}\mathrm{NP} K \mathrm{Mg} \\
\text { (3) }\end{array}$ & $\begin{array}{c}\mathrm{NP} K \mathrm{Kg} \\
+\mathrm{Cu} \\
\text { (4) }\end{array}$ & $\begin{array}{c}\text { NP K Mg } \\
+\quad \mathrm{Zn} \\
(5)\end{array}$ & $\begin{array}{c}\text { NP K Mg } \\
+\underset{+}{\mathrm{Cu}+\mathrm{Zn}} \\
(6)\end{array}$ \\
\hline
\end{tabular}

1) Apports globaux au sol sous forme d'engrais sulfatés. Application en 1975-76 et 1977.

\begin{tabular}{ll} 
(en $\mathrm{kg} / \mathrm{ha}$ ) \\
$\mathrm{Cu}$ & 0 \\
$\mathrm{Zn}$ & 0 \\
\hline
\end{tabular}

2) Exportations par les 8 récoltes de ray-grass anglais (en $\mathrm{kg} / \mathrm{ha}$ )

\begin{tabular}{lcccccc}
$\mathrm{Cu}$ & 0,32 & 0,36 & 0,40 & 0,62 & 0,46 & 0,68 \\
$\mathrm{Zn}$ & 1,77 & 1,93 & 2,35 & 2,38 & 4,60 & 5,00 \\
\hline
\end{tabular}

3) Quantités stockées dans les racines $(0-5 \mathrm{~cm})$

\begin{tabular}{|c|c|c|c|c|c|c|}
\hline $\mathrm{Cu}$ & n.d. & n.d. & 0,09 & 1,14 & 0,11 & 1,52 \\
\hline $\mathrm{Zn}$ & n.d. & n.d. & 0,62 & 0,54 & 1,37 & 2,03 \\
\hline \multicolumn{7}{|c|}{$\begin{array}{l}\text { rées-sorties dans le sol } \\
\text { (en } \mathrm{kg} / \mathrm{ha} \text { ) }\end{array}$} \\
\hline $\mathrm{Cu}$ & $-\quad 0,32$ & $-0,36$ & $-0,49$ & $+21,4$ & $\begin{array}{l}-\quad 0,57 \\
\end{array}$ & $+20,6$ \\
\hline $\mathrm{Zn}$ & $-\quad 1,77$ & $-\quad 1,93$ & $-2,97$ & $-\quad 2,92$ & $+27,90$ & $+26,9$ \\
\hline
\end{tabular}

5) Quantités d'oligo-éléments dans le sol au début de

l'essai

(en $(\mathrm{kg} / \mathrm{ha})$ d'après les analyses

\begin{tabular}{lrrrrr}
$\mathrm{Cu}$ & 3,15 & 3,15 & 3,15 & 3,15 & 3,15 \\
$\mathrm{Zn}$ & 23,00 & 23,00 & 23,00 & 23,00 & 23,00 \\
\hline
\end{tabular}

6) Quantités d'oligo-éléments dans le sol au terme de

(en $\mathrm{kg} / \mathrm{ha}$ ) d'après les analyses

$\begin{array}{lrrrrr}\mathrm{Cu} & 2,68 & 2,82 & 11,80 & 2,60 & 22,70 \\ \mathrm{Zn} & 17,50 & 21,20 & 18,40 & 33,00 & 46,00\end{array}$

7) Bilan global (gains + ou pertes -)

(en $\mathrm{kg} / \mathrm{ha}$ )
$\mathrm{Cu}$
$\mathrm{Zn}$

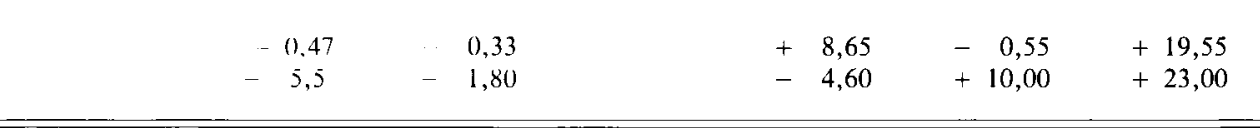

(1)

technique elle-même qui attribue une disponibilité identique pour la plante aux oligo-éléments, qu'ils soient incorporés par la fertilisation cupro-zincique ou présents naturellement dans le sol.

Les analogies avec les résultats des bilans culturaux sont excellentes pour le traitement (2) où les pertes de $\mathrm{Cu}$ et de $\mathrm{Zn}$ sont presque identiques. De même, les pertes ou les gains de $\mathrm{Cu}$ sont très voisins dans les traitements (5) et (6) ; les pertes ou les gains de $\mathrm{Zn}$ des traitements (4) et (6) donnent le sens des phénomènes, appauvrissement ou enrichissement, avec des ordres de grandeurs comparables.

Par contre, de très grandes différences entre les 2 méthodes de calculs apparaissent lorsque $\mathrm{Cu}$ ou $\mathrm{Zn}$ sont apportés seuls. Le bilan de Cu EDTA représente seulement 41 p. 100 de l'excédent du Cu : apportsexportations, tandis que celui du Zn EDTA représente 36 p. 100 du même excédent.

Dans les 2 cas, comme il n'y a apparemment pas de lessivage (fig. 2 et 5), il faut admettre qu'une grande partie de l'élément apporté, $\mathrm{Cu}$ et $\mathrm{Zn}$, a été stockée sous une forme inaccessible à l'extraction par EDTA. Par contre, l'association des 2 fumures $\mathrm{Cu}$ et $\mathrm{Zn}$ permet de maintenir, après 8 ans, une réserve qui représente 95 p. 100 de l'excédent de Cu et 85 p. 100 de celui de $\mathrm{Zn}$.

Dans les parcelles non fertilisées, les teneurs moyennes des plantes en $\mathrm{Cu}$ et $\mathrm{Zn}$ estimées sur 8 campagnes ne sont pas affectées par les traitements $\mathrm{N} P, \mathrm{~N} P \mathrm{Mg}$ et $\mathrm{N} \mathrm{P} \mathrm{K} \mathrm{Mg}$; c'est dire que les quantités totales exportées reflètent principalement le niveau de production lié au traitement.

La fumure $\mathrm{Cu}$ accrô̂t de 55 et 70 p. 100 respectivement les teneurs en $\mathrm{Cu}$ des fourrages en l'absence ou en présence de fertilisation $\mathrm{Zn}$. La fumure $\mathrm{Zn}$ seule accroît les teneurs en $\mathrm{Cu}$ de 15 p. 100 (moyenne sur 8 ans).

La fumure $\mathrm{Zn}$ accroît de 96 et 113 p. 100 respectivement les teneurs en $\mathrm{Zn}$ des fourrages en l'absence ou en présence de fumure $\mathrm{Cu}$. La fertilisation $\mathrm{Cu}$ seule a peu d'influence sur la teneur en $\mathrm{Zn}$. 


\section{CONCLUSION}

Une fertilisation cuprique ou zincique apportée en couverture au sol pendant 3 années consécutives en fin d'hiver, sous forme de sulfates solubles, ne détermine pas un accroissement de production d'un ray-grass anglais. Une fumure $\mathrm{N} \mathrm{P} \mathrm{K} \mathrm{Mg} \mathrm{suffit} \mathrm{à} \mathrm{maintenir} \mathrm{de}$ hauts rendements : 10,4 $\mathrm{t} \mathrm{MS} / \mathrm{ha} /$ an sur 8 ans, très proche du potentiel de production possible à $1000 \mathrm{~m}$ d'altitude sur ces sols volcaniques (NIQUEUX \& ARNAUD, 1975 ; ARNAUd \& NIQUEUX, 1981).

Par contre, la qualité des fourrages, carencés à la fois en $\mathrm{Cu}$ et en $\mathrm{Zn}$ pour satisfaire aux besoins des bovins, est améliorée durablement. Cependant, cette amélioration n'est pas identique pour les 2 oligoéléments.

\section{Enrichissement en cuivre}

Dans le cas du cuivre, les racines constituent un lieu de stockage très important ; la migration vers l'appareil aérien est difficile et très liée à celle de l'azote dans la plante. Les $1^{\text {res }}$ coupes aux stades «début d'épiaison » à « épiaison » donnent une très forte production de matière sèche mais on enregistre un effet de dilution de l'azote, du cuivre et du zinc, simultanément. Il en résulte que les teneurs en $\mathrm{Cu}$ sont insuffisamment corrigées par la fertilisation et restent inférieures au seuil de $7 \mathrm{ppm}$ durant les 3 années d'application de $\mathrm{Cu}$ au sol et celle qui suit les derniers apports. Toutefois, ces teneurs ne cessent d'augmenter, même après la cessation des apports, et dépassent ce seuil dans les $5^{\mathrm{e}}$ et $6^{\mathrm{e}}$ années. La teneur en azote, constitue à la fois un bon indicateur du stade végétatif et des possibilités de migration du $\mathrm{Cu}$ dans les parties aériennes. La fumure cuivrique sous forme de sulfate et une récolte à un stade plus précoce, assurant une teneur en azote supérieure à 2,5 p. 100 de la M.S., apparaissent comme les conditions d'un enrichissement en $\mathrm{Cu}$ suffisant et durable des fourrages de $1^{\text {re }}$ exploitation.

L'influence bénéfique de la fumure associant $\mathrm{Cu}$ et
Zn sur la teneur en $\mathrm{Cu}$ de la $\mathbf{l}^{\text {re }}$ coupe apparaît dès la $1^{\text {re }}$ année de son application et se poursuit durant 3 ans après la cessation des apports. Mais cette évolution est moins régulière sur la $2^{\mathrm{e}}$ coupe et surtout sur la 3 e.

La $2^{\mathrm{e}}$ coupe est encore pauvre en $\mathrm{Cu}$ la $1^{\text {re }}$ année d'application au sol de cet élément, mais suffisamment enrichie les 7 années suivantes.

Quant à la $3^{\mathrm{e}}$ coupe, elle est suffisamment pourvue en $\mathrm{Cu}$ dès la $1^{\mathrm{re}}$ application au sol et reste par la suite à une teneur satisfaisante.

\section{Enrichissement en zinc}

Les fourrages sont suffisamment pourvus en $\mathrm{Zn}$ dès la $2^{\mathrm{e}}$ application au sol de cet élément, et cela pour les 3 coupes annuelles. Par la suite, les teneurs restent supérieures au seuil de carence de $40 \mathrm{ppm}$. L'association des 2 fumures cuivrique et zincique est favorable à une meilleure teneur en $\mathrm{Zn}$ dès la $1^{\mathrm{re}}$ et la $2^{\mathrm{e}}$ année d'application lorsque les fourrages sont encore peu enrichis ; elle permet ainsi de franchir plus rapidement et plus nettement le seuil de carence.

En conclusion, l'application d'une fumure associée de $\mathrm{Cu}$ et $\mathrm{Zn}$ s'avère plus efficace qu'un apport séparé de ces 2 oligo-éléments. Elle permet d'enrichir durablement les fourrages d'un ray-grass anglais cultivé en sol volcanique. L'enrichissement en Zn est plus rapide que celui en $\mathrm{Cu}$. En ce qui concerne ce dernier élément, la récolte de la coupe à un stade assez précoce, encore bien pourvu en azote, est indispensable pour que la teneur en $\mathrm{Cu}$ des fourrages dépasse le seuil de carence de $7 \mathrm{ppm}$.

Reçu le 21 janvier 1985. Accepté le 7 juin 1985.

\section{REMERCIEMENTS}

Les auteurs remercient tout particulièrement le $\mathrm{Dr} C$. Evans de la Hill Farming Research Organization d'Edimbourg pour ses suggestions et conseils dans l'interprétation des résultats.

\section{RÉFÉRENCES BIBLIOGRAPHIQUES}

\begin{abstract}
Arnaud R., Niqueux M., 1981. Bilan de quinze années d'expérimentation sur les espèces et variétés fourragères en altitude dans le Massif Central (Laqueuille et Bourg-Lastic). Fourrages, 87, 3-52.
\end{abstract}

Balland D., 1983. Sur céréales, le cuivre peut valoir de l'or. Perspectives agricoles, 66, 1-16.

Bellanger J., 1971. Dosage des oligo-éléments dans les fourrages. Ann. Nutr. Alim., 25, 359-396.

Bellanger J., Perigaud S., Lamand M., 1973. Carences en oligoéléments en France. III - Eléments d'enquête obtenus par les analyses de fourrages. Ann. Rech. Vétér., 4, 565-598.

Coic Y., Lesaint C., Cholet Y., 1974. Accumulation comparée du cuivre dans les racines et parties aériennes des plantes fourragères en fonction de l'alimentation en cuivre et de la nature nitrique ou nitrico-ammoniacale de l'alimentation azotée. C. R. Acad. Agric. Fr., 60, 1162-1171.

Coppenet M., 1970a. Les oligo-éléments en France. Exemples de problèmes régionaux. 1. Le Massif armoricain. Ann. Agron., 21, 5, 587-601.

Coppenet M., 1970b. Effet des sels de cuivre et de cobalt sur l'enri- chissement des plantes fourragères en ces deux oligo-éléments. C. $R$. Acad. Agric. Fr., 56, 923-937.

Coppenet M., Simon J. C., 1984. Sur les teneurs en éléments minéraux des graminées fourragères. Possibilités d'amélioration génétique. Fourrages, 97, 17-33.

Dartigues A., 1964. Les déficiences en zinc chez les végétaux et leurs causes. Ann. Agron., 15, 667-691.

Dejou J., de Montard F. X., 1982. Effet positif des fertilisations potassique et magnésienne dans les sols volcaniques du Massif Central. Dossier $K^{2} O, S C P A$ Mulhouse, 21, 13-24.

Dejou J., de Montard F. X., Lamand M., Bellanger J., 1980. Déficiences en cuivre et en zinc observées en zones volcaniques de l'Auvergne ; possibilité d'amélioration de la qualité des foins par apport au sol de ces deux oligo-éléments. Fourrages, 84, 69-85.

Jarvis S. C., 1981. The uptake and distribution of copper in some forage grasses as affected by nitrate-nitrogen supply in flowing solution culture. Ann. Bot., 48, 147-157.

Lamand M., 1970. Carences en oligo-éléments chez les ruminants. Cah. Méd. Vét., 2, 1-16. 
Lamand M., 1971. Diagnostic des carences en oligo-éléments chez l'animal. Ann. Nutr. Alim., 25, B. 379-B. 410.

Lamand M., 1976. Les carences en oligo-éléments en France. Trait d'Union Agric., 62.

Lamand M., Gueguen L., 1978. Eléments minéraux majeurs et oligo-éléments (chap. 4) in Publ. INRA, Alimentation des Ruminants, $621 \mathrm{p}$.

Lamand M., Perigaud S., 1973. Carences en oligo-éléments chez les ruminants en France. I - Eléments d'enquête obtenus dans la pratique vétérinaire. Ann. Rech. Vét., 4, 513-534.

Loneragan J. F., Robson A. D., Graham R. D., 1981. Copper in soils and plants. Acad. Press Australia, $380 \mathrm{p}$.

De Montard F. X., Perigaud S., 1975. Influence de quelques facteurs naturels et des facteurs d'intensification sur la composition en oligo-éléments des fourrages de prairie permanente. Oligo-éléments, $N^{\circ}$ spécial du Bull. Tech. du CRZV de Theix, 120-136.

Niqueux M., Arnaud R., 1975. Comportement des espèces et variétés fourragères en montagne. Fourrages, 64, 89-102.
Owen Jr C. A., 1981. Biological aspects of copper. Occurrence, assay and interrelationships. In « Copper in Biology and Medecine séries », Noyes Publ. Park Ridge, New-Jersey, 139 p.

Perigaud S., 1970. Les carences en oligo-éléments chez les ruminants en France. Leur diagnostic, les problèmes soulevés par l'intensification fourragère. Ann. Agron., 21, 635-669.

Perigaud S., Lamand M., 1973. Carences en oligo-éléments chez les ruminants en France. II - Eléments d'enquête obtenus dans les élevages. Ann. Rech. Vét., 4, 535-563.

Simon J.-C., Le Corre L., Coppenet M., 1983. Essai comparatif de dix graminées fourragères dont six cultivars de brome dans le Finistère. Production, valeur alimentaire, composition minérale. Fourrages, 93, 85-108.

Trichet J., 1983. Rétention des métaux lourds ( $\mathrm{Cu}, \mathrm{Mn}, \mathrm{Zn}, \mathrm{Pb}$, $\mathrm{Cd}, \mathrm{Ni}, \mathrm{Co}, \mathrm{As}$ ) par les sols volcaniques. Incidences sur le transfert de ces métaux par les plantes, le bétail et les eaux. $C$. $R$. Contrat de recherche SDS 81-219. Minist. Environment et Labo-Geol. Appliquée Univ. Orléans, $29 \mathrm{p}$. 